\title{
Are There Changes in the Fatty Acid Profile of Breast Milk with Supplementation of Omega-3 Sources? A Systematic Review
}

\section{Existem mudanças no perfil de ácidos graxos no leite materno com a suplementação de fontes de ômega 3? Uma revisão sistemática}

\author{
Yasmin Notarbartolo di Villarosa do Amaral ${ }^{1}$ Daniele Marano ${ }^{1}$ Leila Maria Lopes da Silva ${ }^{1}$ \\ Aline Carnevale Lia Dias Guimarães ${ }^{1} \quad$ Maria Elisabeth Lopes Moreira ${ }^{1}$ \\ ${ }^{1}$ Clinical Research Unit, Instituto Nacional de Saúde da Mulher, da \\ Criança e do Adolescente Fernandes Figueira, Fundação Oswaldo \\ Cruz, Rio de Janeiro, RJ, Brazil \\ Rev Bras Ginecol Obstet 2017;39:128-141. \\ Address for correspondence Yasmin Notarbartolo di Villarosa do \\ Amaral, MSc, Fundação Oswaldo Cruz, Instituto Nacional de Saúde da \\ Mulher, da Criança e do Adolescente Fernandes Figueira, Unidade de \\ Pesquisa Clínica, Av. Rui Barbosa, 716, Flamengo, 22250-020, Rio de \\ Janeiro, RJ, Brasil (e-mail: yasminamaral@hotmail.com).
}

\begin{abstract}
Keywords

- pregnant women

- breastfeeding

- human milk

- omega-3 fatty acids

- systematic review
\end{abstract}

Resumo
Purpose To evaluate the effect of supplementation with omega-3 sources on the fatty acid composition of human milk.

Methods The review consisted of the search for articles published in PubMed, Biblioteca Virtual de Saúde (Virtual Health Library[VHL]) and Web of Science databases using the following keywords: fatty acids, omega-3, human milk and supplementation; for this purpose, we have used the program of research to integrate the services for the maintenance of autonomy (PRISMA) checklist. The following selection criteria were used: articles in English, Portuguese, Spanish or Italian, published between 2000 and 2015, and about studies performed in humans. We found 710 articles that met the established criteria; however, only 22 of them were selected to be part of this study. Results All studies found a positive relationship between the consumption of omega3 sources and their concentration in human milk. The differences in the findings are due to the distinct methods used, such as the specific time of the omega- 3 supplementation, the type of omega-3 source offered, as well as the sample size.

Conclusion Although the studies were different in several methodological aspects, it was possible to observe the importance of omega-3 supplementation during gestation and/or the puerperium.

Objetivo Avaliar o efeito da suplementação com fontes de ômega 3 sobre a composição de ácidos graxos do leite humano. received

February 11, 2016

accepted

December 20, 2016

published online

March 17, 2017
DOI http://dx.doi.org/

10.1055/s-0037-1599094. ISSN 0100-7203.
Copyright $\odot 2017$ by Thieme-Revinter

Publicações Ltda, Rio de Janeiro, Brazil
License terms

(c) $(1) \$$ 


\section{Palavras-chave}

- gestantes

- aleitamento materno

- leite humano

- ácidos graxos ômega-3

- revisão sistemática
Métodos A revisão consistiu na busca de artigos publicados nas bases de dados PubMed, Biblioteca Virtual de Saúde e Web of Science utilizando-se as palavras-chave: ácidos graxos, ômega-3, leite materno e suplementação; para isso, foi utilizado o checklist PRISMA. Foram utilizados os seguintes critérios para a seleção: artigos publicados em inglês, português, espanhol ou italiano, entre os anos de 2000 a 2015, sobre estudos realizados em humanos. A busca bibliográfica, segundo a estratégia estabelecida, resultou em 710 artigos. Entretanto, apenas 22 destes foram selecionados para compor a presente revisão.

Resultados Todos os estudos encontraram relação positiva entre o consumo de fontes de ômega 3 e sua concentração no leite humano. As diferenças nos achados se devem aos métodos empregados como, por exemplo, o momento da suplementação do ômega 3, o tipo de fonte de ômega 3 ofertado, e o tamanho amostral.

Conclusão Apesar de os estudos serem díspares em inúmeros aspectos metodológicos, observou-se a importância da suplementação do ômega 3 na gestação e/ou no puerpério.

\section{Introduction}

The importance of polyunsaturated fatty acids of the omega$3(\omega-3)$ series (docosahexaenoic acid [22:6 $\omega 3$, DHA] and eicosapentaenoic acid [20:5 $\omega 3, E P A]$ ) in the development of the fetal brain, as well as in the cognitive and visual acuity of the child, is widely recognized. These fatty acids are part of the composition of the cell membranes and the nervous system, especially DHA, which is preferentially transported by the placenta to the fetus and provides important components to the phospholipid membrane. ${ }^{1,2}$

The amount of fatty acids in human milk (HM) depends on maternal stocks, dietary intake and synthesis thereof in the mammary glands. ${ }^{3}$ The concentration of DHA varies specifically, probably due to the woman's feeding habits, since its synthesis in the mammary gland is minimal. ${ }^{1,2,4}$ During gestation and lactation, this synthesis is limited by the fetus. For this reason, numerous studies have been conducted to evaluate the effects of the supplementation of this fatty acid on the composition of $\mathrm{HM} .^{4,5}$ Other facts to be taken into consideration are that the concentration of DHA in HM decreases as lactation progresses, and that supplementation during lactation raises DHA concentrations in breast milk. ${ }^{6}$

The study conducted by Bortolozo et al $(2013)^{2}$ aimed to evaluate the impact of omega-3 fatty acid supplementation between the third trimester of pregnancy and the third month after delivery, and its influence on the composition of HM. Although no statistical difference was found in the total lipid values between the studied groups, the milk of mothers supplemented with fish oil had higher concentrations of DHA and EPA, demonstrating that a higher consumption of omega-3 may influence its concentration in HM. ${ }^{2}$ However, the results on the effects of omega-3 supplementation during gestation are still contradictory. 4

Due to the controversies between the studies, as well as to the importance of the theme for the health of the newborn, this systematic review aims to evaluate the studies that verified the effects of omega-3 supplementation during pregnancy and/or the puerperium on the composition of
HM. The bibliographical survey of this theme aims to assist the maternal and infant populations, together with health professionals, to determine the importance of supplementation, offering subsidies for its practice.

\section{Methods}

A systematic review of the available literature consisted of a retrospective search of scientific articles that aimed to evaluate the composition of HM after supplementation with omega-3 fatty acids.

The following bibliographic databases have been used: PubMed, Biblioteca Virtual da Saúde (Virtual Health Library [VHL]) and Web of Science. The search for the articles was performed independently by two researchers, and it began in August and ended in October of 2015. The selected studies were published during the period comprised between 2000 and 2015. The following keywords were used in the search strategy: fatty acids, omega-3, human milk and supplementation.

The bibliographic search was performed according to the established strategy, and resulted in 710 articles. A total of 163 articles were found in the VHL database; however, after reading the abstracts, we have selected 11 thereof; 239 articles were found in the PubMed database; however, we have only selected 21 of these; and 308 articles were found in the Web of Science database, from which we have selected 2 articles. Thus, a total of 22 articles have been selected to compose the present study, reiterating that there were 12 articles replicated in the analyzed databases. The others were suppressed for the following reasons: discussion of different associations between omega-3 and HM, such as allergy, visual acuity and growth; literature reviews; studies replicated across different databases; studies published in other languages and/or that were were not available in their entirety. We used a checklist with 27 items and a 4-step flowchart, advocated by PRISMA, ${ }^{7}$ which aims to help authors improve the reporting of systematic reviews. 


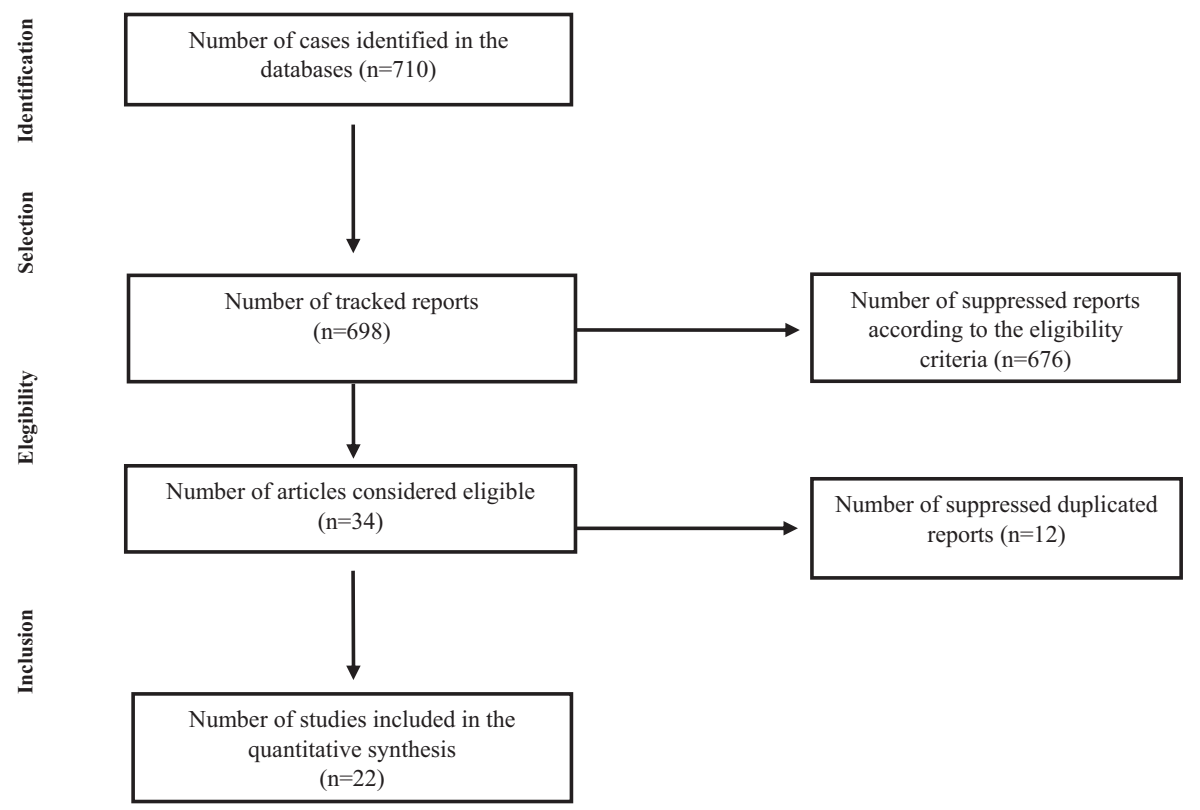

Fig. 1 Flowchart of the selection process for selected articles - PRISMA.

Therefore, a summary of each stage of the selection process of articles that composed this systematic review was arranged in the Flowchart (- Fig. 1).

The criteria used in the selection of articles for the review included language (Portuguese, English, Spanish and Italian) and year of publication (2000 to 2015).

The selected articles were compared in relation to the following parameters: year of publication, country of origin, sample size, average age of participants, type of design, rate of follow-up losses, period in which the woman and the milk have been evaluated, omega-3 supplementation period, type of supplement, amount offered, omega-3 evaluation methods, confounding factors controlled in the analysis, estimators used in the statistical analysis, and main results observed.

\section{Results}

Once the established strategy was put into practice, 22 articles were selected by bibliographic search to compose the present revision. Four were originated in the United States, one in Canada, one in Denmark, three in Brazil, three in Australia, three in Chile, one in Israel, one in Iceland, one in Mexico, two in Germany, and two in the Netherlands. Regarding the age groups, the majority of the articles (18) only reported the average age of the participants. Regarding language, two articles were written in Spanish, one in Portuguese and the remaining ones in English (-Table 1).

As for the design employed, intervention studies were used in most cases, and there was only one observational study. Information on follow-up losses were obtained from 21 studies. The losses ranged from 0 to $78 \%$. The results among the studies, regarding the period of evaluation of the woman and the milk, were quite dissimilar. Regarding the period of supplementation, it was observed that 12 studies evaluated supplementation in infants, 7 in pregnant women, and only 3 evaluated supplementation both in pregnancy and in the puerperium (-Table $\mathbf{2}$ ).

In regards to the type of supplement or food offered to the participants, 3 studies used dairy supplements, 16 provided DHA-rich oils (such as, tuna, single-celled algae, cod, flaxseed), 2 supplied fish (sardine and jure) and 1 study performed food education to increase the consumption of fish sources. As for the method to evaluate the amount of omega 3 in HM, it was observed that all the studies used chromatography. The DHA value ranged from $170 \mathrm{mg}$ to $2,200 \mathrm{mg}$ per day ( - Table 3 ).

Among the 22 selected articles, 9 did not evaluate the consumption of omega-3 food sources. Among those who reported it, the majority (32\%) used the food frequency questionnaire (FFQ). Regarding the estimators, it was observed that nine studies used the average, seven used the correlation index, five the median and only one study used the combination of correlation index with the average. Regarding the exclusion criteria, nine articles did not mention them in the methods ( - Table 4 ).

Regarding supplementation, all studies found a positive relationship between the consumption of omega- 3 sources and their concentration in HM, therefore, highlighting the importance of supplementation of these fatty acids during pregnancy and/or puerperium, which translates into positive results in both cognitive development and visual acuity.

\section{Discussion}

The omega- 3 and omega- 6 polyunsaturated fatty acids consumed through dietary triglycerides are digested in the small intestines and can then be absorbed, transported into the bloodstream and taken up between tissues throughout the body (including brain, retina and heart). ${ }^{8}$ Essential dietary 
Are There Changes in the Fatty Acid of Breast Milk with Supplementation of Omega-3? Amaral et al. 131

Table 1 Year of publication, origin, sample size, and age of the participants of the selected studies on the fatty acid profile of breast milk with the supplementation of omega-3 sources, 2000-2015

\begin{tabular}{|c|c|c|c|c|}
\hline Authors & Pub year & Country & Sample (n) & Age (years) \\
\hline Atalah et $\mathrm{al}^{5}$ & 2009 & Chile & $\begin{array}{l}352 \text { pregnant } \\
\text { women }\end{array}$ & $\begin{array}{l}\text { Intervention } \\
\text { group } 26.7 \text { and } \\
\text { control group } \\
25.0 \text { (average) }\end{array}$ \\
\hline Bergmann et al ${ }^{38}$ & 2008 & Germany & $\begin{array}{l}144 \text { pregnant } \\
\text { women }\end{array}$ & 30.7 (average) \\
\hline Boris et $\mathrm{al}^{27}$ & 2004 & Denmark & $\begin{array}{l}44 \text { pregnant/ } \\
\text { puerperal women }\end{array}$ & $\mathrm{NI}$ \\
\hline Bortolozo et $\mathrm{al}^{2}$ & 2013 & Brazil & $\begin{array}{l}80 \text { pregnant/ } \\
\text { puerperal women }\end{array}$ & 25.0 (average) \\
\hline Dunstan et al ${ }^{20}$ & 2007 & Australia & $\begin{array}{l}98 \text { pregnant } \\
\text { women }\end{array}$ & $\mathrm{NI}$ \\
\hline Fidler et al $^{17}$ & 2000 & Germany & $\begin{array}{l}10 \text { puerperal } \\
\text { women }\end{array}$ & 30.6 (average) \\
\hline Francois et $\mathrm{al}^{36}$ & 2003 & United States & $\begin{array}{l}9 \text { puerperal } \\
\text { women }\end{array}$ & 28.0 to 39.0 \\
\hline $\begin{array}{l}\text { Gaete and } \\
\text { Atalah }\end{array}$ & 2003 & Chile & $\begin{array}{l}26 \text { puerperal } \\
\text { women }\end{array}$ & 26.9 (average) \\
\hline $\begin{array}{l}\text { Gaete, Atalah and } \\
\text { Araya } 31\end{array}$ & 2002 & Chile & $\begin{array}{l}28 \text { puerperal } \\
\text { women }\end{array}$ & $\begin{array}{l}\text { Intervention } \\
\text { group } 25.6 \text { and } \\
\text { control group } \\
26.4 \text { (average) }\end{array}$ \\
\hline van Goor et al ${ }^{39}$ & 2009 & Netherlands & $\begin{array}{l}182 \text { pregnant/ } \\
\text { puerperal women }\end{array}$ & 32.4 (average) \\
\hline Hawkes et al ${ }^{40}$ & 2001 & Australia & $\begin{array}{l}120 \text { puerperal } \\
\text { women }\end{array}$ & 30.2 (average) \\
\hline $\begin{array}{l}\text { Imhoff-Kunsch } \\
\text { et al }\end{array}$ & 2011 & Mexico & $\begin{array}{l}1,094 \text { pregnant } \\
\text { women }\end{array}$ & 26.0 (average) \\
\hline Jensen et $\mathrm{al}^{18}$ & 2000 & United States & $\begin{array}{l}26 \text { puerperal } \\
\text { women }\end{array}$ & 29.2 (average) \\
\hline Marc et $\mathrm{al}^{32}$ & 2011 & Canada & $\begin{array}{l}32 \text { puerperal } \\
\text { women }\end{array}$ & $\begin{array}{l}\text { Intervention } \\
\text { group } 27.2 \text { and } \\
\text { control group } \\
26.9 \text { (average) }\end{array}$ \\
\hline Olafsdottir et al ${ }^{41}$ & 2006 & Iceland & $\begin{array}{l}77 \text { puerperal } \\
\text { women }\end{array}$ & 31.0 (average) \\
\hline Patin et $\mathrm{al}^{35}$ & 2006 & Brazil & $\begin{array}{l}31 \text { puerperal } \\
\text { women }\end{array}$ & 27.9 (average) \\
\hline Ribeiro et $\mathrm{al}^{30}$ & 2012 & Brazil & $\begin{array}{l}51 \text { pregnant } \\
\text { women }\end{array}$ & 20.0 to 30.0 \\
\hline Sherry et $\mathrm{al}^{42}$ & 2015 & United States & $\begin{array}{l}89 \text { pregnant } \\
\text { women }\end{array}$ & 29.0 (average) \\
\hline Smit et $\mathrm{al}^{37}$ & 2000 & Israel & $\begin{array}{l}26 \text { puerperal } \\
\text { women }\end{array}$ & 23.5 (average) \\
\hline Smithers et al ${ }^{43}$ & 2010 & Australia & $\begin{array}{l}121 \text { puerperal } \\
\text { women }\end{array}$ & 30.0 (average) \\
\hline Valentine et $\mathrm{al}^{44}$ & 2013 & United States & $\begin{array}{l}21 \text { puerperal wo- } \\
\text { men (donors) }\end{array}$ & 31.0 (average) \\
\hline Weseler et al ${ }^{45}$ & 2008 & Netherlands & $\begin{array}{l}52 \text { pregnant/ } \\
\text { puerperal women }\end{array}$ & 31.7 (average) \\
\hline
\end{tabular}

Abbreviation: NI, Not informed. 
132 Are There Changes in the Fatty Acid of Breast Milk with Supplementation of Omega-3? Amaral et al.

Table 2 Type of design, losses, period of evaluation and supplementation of sources of omega-3, 2000-2015

\begin{tabular}{|c|c|c|c|c|c|}
\hline Authors & Design & Losses (\%) & $\begin{array}{l}\text { Period in which the } \\
\text { women were evalu- } \\
\text { ated }\end{array}$ & $\begin{array}{l}\text { Period in which the milk } \\
\text { was evaluated }\end{array}$ & $\begin{array}{l}\text { Period of sup- } \\
\text { plementation } \\
\text { of omega-3 } \\
\text { fatty acids }\end{array}$ \\
\hline Atalah et $\mathrm{al}^{5}$ & Clinical trial & 70.0 & $\begin{array}{l}\text { Three times during } \\
\text { gestation and once } \\
\text { in the second month } \\
\text { after delivery }\end{array}$ & 2nd month after childbirth & $\begin{array}{l}\text { During } \\
\text { pregnancy }\end{array}$ \\
\hline $\begin{array}{l}\text { Bergmann } \\
\text { et } \mathrm{al}^{38}\end{array}$ & $\begin{array}{l}\text { Randomized } \\
\text { double blind } \\
\text { clinical trial }\end{array}$ & 45.8 & $\begin{array}{l}21 \text { st and } 37 \text { th weeks } \\
\text { of gestation, at de- } \\
\text { livery, and } 1 \text { and } \\
3 \text { months after } \\
\text { delivery }\end{array}$ & 3rd month after childbirth & $\begin{array}{l}21 \text { st to } 37 \text { th } \\
\text { week, conti- } \\
\text { nuation was } \\
\text { optional }\end{array}$ \\
\hline Boris et $\mathrm{al}^{27}$ & $\begin{array}{l}\text { Randomized } \\
\text { clinical trial }\end{array}$ & 18.2 & $\begin{array}{l}\text { 30th gestational } \\
\text { week and the } 1 \mathrm{st} \\
\text { month after delivery }\end{array}$ & $\begin{array}{l}\text { 4th, } 16 \text { th and 30th days } \\
\text { after childbirth }\end{array}$ & $\begin{array}{l}\text { From the 30th } \\
\text { gestational } \\
\text { week until de- } \\
\text { livery (group 1) } \\
\text { or until the } \\
\text { 30th day after } \\
\text { delivery }\end{array}$ \\
\hline Bortolozo et $\mathrm{al}^{2}$ & $\begin{array}{l}\text { Randomized } \\
\text { controlled clini- } \\
\text { cal trial }\end{array}$ & 25.0 & $\begin{array}{l}\text { Last trimester of } \\
\text { pregnancy until the } \\
\text { 3rd month of } \\
\text { lactation }\end{array}$ & $\begin{array}{l}\text { 30th, 60th, 90th days after } \\
\text { childbirth }\end{array}$ & $\begin{array}{l}\text { Last trimester } \\
\text { of pregnancy } \\
\text { (baseline) until } \\
\text { the 3rd month } \\
\text { of lactation }\end{array}$ \\
\hline Dunstan et $\mathrm{al}^{20}$ & $\begin{array}{l}\text { Randomized } \\
\text { double blind } \\
\text { clinical trial }\end{array}$ & 25.0 & $\begin{array}{l}3^{\text {rd }} \text { and } 6 \text { th days, and } \\
6 \text { th month after } \\
\text { childbirth }\end{array}$ & $\begin{array}{l}3^{\text {rd }} \text { and } 6 \text { th days, and } 6 \text { th } \\
\text { month after childbirth }\end{array}$ & $\begin{array}{l}\text { 20th week of } \\
\text { gestation until } \\
\text { delivery }\end{array}$ \\
\hline Fidler et al ${ }^{17}$ & $\begin{array}{l}\text { Randomized } \\
\text { clinical trial }\end{array}$ & 0.0 & $\begin{array}{l}\text { From the 4th week } \\
\text { until the 6th after } \\
\text { delivery }\end{array}$ & $\begin{array}{l}\text { In the 4th week after deliv- } \\
\text { ery (before starting supple- } \\
\text { mentation), at the 6th week } \\
\text { after delivery (after supple- } \\
\text { mentation), and at } 6,12,24 \text {, } \\
36 \text { and } 48 \text { hours after intake } \\
\text { of the supplement }\end{array}$ & $\begin{array}{l}\text { From } 4 \text { to } \\
6 \text { weeks after } \\
\text { delivery ( } 14 \\
\text { days) }\end{array}$ \\
\hline Francois et $\mathrm{al}^{36}$ & Clinical trial & 22.0 & $\begin{array}{l}10 \text { weeks: } 2 \text { weeks of } \\
\text { washout* at baseline } \\
\text { (to stabilize the } \\
\text { omega- } 6 \text { and } 3 \text { in- } \\
\text { takes); } 4 \text { weeks of } \\
\text { linseed oil supple- } \\
\text { mentation and } \\
4 \text { weeks after } \\
\text { supplementation }\end{array}$ & $\begin{array}{l}1 \text { sample at baseline, } 1 \\
\text { sample after washout* ( } 2 \\
\text { weeks after the start of } \\
\text { study), } 4 \text { samples at weekly } \\
\text { intervals during } 4 \text { weeks of } \\
\text { supplementation, and } 4 \\
\text { samples at weekly intervals } \\
\text { during the post-supplemen- } \\
\text { tation period ( } 4 \text { weeks). }\end{array}$ & 4 weeks \\
\hline $\begin{array}{l}\text { Gaete and } \\
\text { Atalah }^{1}\end{array}$ & Cohort & 7.69 & $\begin{array}{l}\text { From entry into the } \\
\text { study until the } 2 \text { nd } \\
\text { week after food } \\
\text { education }\end{array}$ & $\begin{array}{l}2 \text { weeks after food } \\
\text { education }\end{array}$ & $\begin{array}{l}\text { Food education } \\
\text { on the day of } \\
\text { study entry }\end{array}$ \\
\hline Gaete et $\mathrm{al}^{31}$ & $\begin{array}{l}\text { Randomized } \\
\text { clinical trial }\end{array}$ & 17.2 & $\begin{array}{l}\text { From the study entry } \\
\text { to the } 15 \text { th day after } \\
\text { intervention }\end{array}$ & $\begin{array}{l}15 \text { days after } \\
\text { intervention }\end{array}$ & 15 days \\
\hline van Goor et al ${ }^{39}$ & $\begin{array}{l}\text { Randomized } \\
\text { double blind } \\
\text { clinical trial }\end{array}$ & 51.6 & $\begin{array}{l}\text { Registration day up } \\
\text { to } 12 \text { weeks after } \\
\text { delivery }\end{array}$ & $\begin{array}{l}\text { 2nd to } 12 \text { th weeks } \\
\text { after delivery }\end{array}$ & $\begin{array}{l}\text { 17th week of } \\
\text { gestation until } \\
\text { the } 12 \text { th week } \\
\text { after childbirth }\end{array}$ \\
\hline Hawkes et al ${ }^{40}$ & $\begin{array}{l}\text { Randomized } \\
\text { double blind } \\
\text { clinical trial }\end{array}$ & 31.7 & $\begin{array}{l}\text { 3rd day after birth } \\
\text { until the end of the } \\
\text { 12th week after } \\
\text { delivery }\end{array}$ & $\begin{array}{l}\text { In the } 4 \text { th week after } \\
\text { delivery }\end{array}$ & 12 weeks \\
\hline
\end{tabular}


Table 2 (Continued)

\begin{tabular}{|c|c|c|c|c|c|}
\hline Authors & Design & Losses (\%) & $\begin{array}{l}\text { Period in which the } \\
\text { women were evalu- } \\
\text { ated }\end{array}$ & $\begin{array}{l}\text { Period in which the milk } \\
\text { was evaluated }\end{array}$ & $\begin{array}{l}\text { Period of sup- } \\
\text { plementation } \\
\text { of omega-3 } \\
\text { fatty acids }\end{array}$ \\
\hline $\begin{array}{l}\text { Imhoff-Kunsch } \\
\text { et al }\end{array}$ & $\begin{array}{l}\text { Randomized } \\
\text { double blind } \\
\text { clinical trial }\end{array}$ & 11.0 & $\begin{array}{l}\text { Gestation (18th to } \\
22 \text { nd weeks) until } \\
1 \text { month after } \\
\text { delivery }\end{array}$ & 1 month after birth & $\begin{array}{l}\text { From the } 18 \text { th } \\
\text { to the } 22 \text { nd ge- } \\
\text { stational week } \\
\text { until delivery }\end{array}$ \\
\hline Jensen et al $^{18}$ & Clinical trial & 7.7 & $\begin{array}{l}\text { 2nd to 8th weeks } \\
\text { after delivery }\end{array}$ & $\begin{array}{l}\text { At the } 2 \mathrm{nd}, 5 \text { th and } 8 \text { th } \\
\text { weeks after delivery }\end{array}$ & 6 weeks \\
\hline Marc et $\mathrm{al}^{32}$ & Clinical trial & 25.0 & $\begin{array}{l}1 \text { postnatal week } \\
\text { (between } 3 \text { and } 7 \\
\text { days) before starting } \\
\text { DHA supplementa- } \\
\text { tion and at follow-up } \\
\text { at } 15 \text { days ( } 3 \text { weeks) } \\
\text { and } 49 \text { days ( } 7 \\
\text { weeks) }\end{array}$ & $\begin{array}{l}\text { First postnatal week (be- } \\
\text { tween } 3 r d \text { and } 7 \text { th days) } \\
\text { before starting supplemen- } \\
\text { tation and at follow-up on } \\
\text { days } 15 \text { and } 49\end{array}$ & $\begin{array}{l}1 \text { week after } \\
\text { delivery until } \\
\text { term ( } 36 \\
\text { weeks) - }>8- \\
12 \text { weeks of } \\
\text { supplementa- } \\
\text { tion }\end{array}$ \\
\hline $\begin{array}{l}\text { Olafsdottir } \\
\text { et } \mathrm{al}^{41}\end{array}$ & $\begin{array}{l}\text { Randomized } \\
\text { clinical trial }\end{array}$ & 48.0 & $\begin{array}{l}\text { 2nd and 4th months } \\
\text { after delivery }\end{array}$ & $\begin{array}{l}\text { 2nd and 4th months after } \\
\text { delivery }\end{array}$ & $\begin{array}{l}\text { Registration } \\
\text { day up to } \\
4 \text { months after } \\
\text { delivery }\end{array}$ \\
\hline Patin et $\mathrm{al}^{35}$ & Clinical trial & $\mathrm{NI}$ & $\begin{array}{l}0,15 \text { and } 30 \text { days } \\
\text { after delivery }\end{array}$ & $\begin{array}{l}0,15 \text { and } 30 \text { days after } \\
\text { delivery }\end{array}$ & $\begin{array}{l}\text { 1st and 15th } \\
\text { days after } \\
\text { delivery }\end{array}$ \\
\hline Ribeiro et $\mathrm{al}^{30}$ & $\begin{array}{l}\text { Randomized } \\
\text { clinical trial }\end{array}$ & 78.4 & $\begin{array}{l}\text { 30th gestational } \\
\text { week up to } 15 \text { days } \\
\text { after delivery }\end{array}$ & 15th day after delivery & $\begin{array}{l}15 \text { days (from } \\
\text { the } 30 \text { th gesta- } \\
\text { tional week) }\end{array}$ \\
\hline Sherry et al ${ }^{42}$ & Clinical trial & 7.9 & $\begin{array}{l}\text { From enrollment } \\
\text { (4th to 6th weeks } \\
\text { after delivery) up to } \\
6 \text { weeks after } \\
\text { supplementation }\end{array}$ & $\begin{array}{l}\text { Baseline and 6th week } \\
\text { aftersupplementation }\end{array}$ & 6 weeks \\
\hline Smit et $\mathrm{al}^{37}$ & Clinical trial & 11.0 & For one week & $\begin{array}{l}\text { Baseline shortly after inges- } \\
\text { tion of the supplement. On } \\
\text { day } 1 \text { and day } 7 \text { after the } \\
\text { intake of the supplement }\end{array}$ & 1 week \\
\hline Smithers et al ${ }^{43}$ & $\begin{array}{l}\text { Randomized } \\
\text { double blind } \\
\text { clinical trial }\end{array}$ & 19.0 & $\begin{array}{l}\text { During all hospitali- } \\
\text { zation of the pre- } \\
\text { term newborn }\end{array}$ & $\begin{array}{l}\text { At intervals of } 2 \text { weeks dur- } \\
\text { ing the hospitalization of } \\
\text { the newborn }\end{array}$ & $\begin{array}{l}\text { From study en- } \\
\text { try (delivery } \\
<33 \text { weeks) } \\
\text { until the ex- } \\
\text { pected date of } \\
\text { delivery }\end{array}$ \\
\hline $\begin{array}{l}\text { Valentine } \\
\text { et } \mathrm{al}^{44}\end{array}$ & $\begin{array}{l}\text { Randomized } \\
\text { clinical trial }\end{array}$ & 38.0 & $\begin{array}{l}3 \text { days before sup- } \\
\text { plementation until } \\
12 \text { weeks post } \\
\text { supplementation }\end{array}$ & $\begin{array}{l}0,7 \text { th, } 14 \text { th, } 21 \text { st, } 28 \text { th and } \\
84 \text { th days after the } \\
\text { supplementation }\end{array}$ & $\begin{array}{l}\text { During all the } \\
\text { time they do- } \\
\text { nated milk to } \\
\text { the milk bank } \\
\text { (from 7-90 } \\
\text { days) }\end{array}$ \\
\hline Weseler et al ${ }^{45}$ & $\begin{array}{l}\text { Randomized } \\
\text { double blind } \\
\text { clinical trial }\end{array}$ & 34.6 & $\begin{array}{l}\text { Pregnancy ( } 36 \\
\text { weeks) up to the } \\
11 \text { th week after } \\
\text { delivery }\end{array}$ & $\begin{array}{l}3 \text { rd, } 5 \text { th and } 11 \text { th weeks } \\
\text { after delivery }\end{array}$ & $\begin{array}{l}\text { Gestation (36th } \\
\text { week) up to } \\
11 \text { th week } \\
\text { postpartum }\end{array}$ \\
\hline
\end{tabular}

Abbreviation: NI, not informed.

Note: *Washout: time necessary for the concentration of a medicinal product to be negligible after cessation of therapy. 
134 Are There Changes in the Fatty Acid of Breast Milk with Supplementation of Omega-3? Amaral et al.

Table 3 Characteristics of the selected studies on the profile of fatty acids in breast milk with omega-3 sources supplementation, 2000-2015

\begin{tabular}{|c|c|c|c|c|c|}
\hline Authors & $\begin{array}{l}\text { Type of } \\
\text { supplement/food } \\
\text { used as source of } \\
\text { omega-3 }\end{array}$ & Amount offered & Placebo/control & $\begin{array}{l}\text { Method used to } \\
\text { evaluate omega-3 } \\
\text { intake }\end{array}$ & $\begin{array}{l}\text { Method to evalu- } \\
\text { ate the amount } \\
\text { of omega-3 pre- } \\
\text { sent in HM }\end{array}$ \\
\hline Atalah et $\mathrm{al}^{5}$ & $\begin{array}{l}\text { Milk drink made } \\
\text { from powdered } \\
\text { milk and hydro- } \\
\text { lyzed cereals en- } \\
\text { riched with } \\
\text { microencapsula- } \\
\text { ted vitamins, } \\
\text { minerals and } \\
\text { omega-3 fatty } \\
\text { acids }\end{array}$ & $\begin{array}{l}60 \mathrm{mg} \text { DHA }+14 \\
\mathrm{mg} \text { EPA in } 200 \mathrm{~mL} \\
(2 \mathrm{~kg} / \text { month })\end{array}$ & Powdered milk & Food survey & Chromatography \\
\hline Bergmann et $a^{38}$ & $\begin{array}{l}\text { Supplement based } \\
\text { on acidified and } \\
\text { flavored milk }\end{array}$ & $\begin{array}{l}\text { Group 1: Basic } \\
\text { supplement plus } \\
4.5 \mathrm{~g} \text { FOS } \\
\text { Group 2: Basic } \\
\text { supplement with } \\
\text { FOS }+200 \mathrm{mg} \text { of } \\
\text { DHA }\end{array}$ & $\begin{array}{l}\text { Basic supplement } \\
\text { enriched with vi- } \\
\text { tamins and } \\
\text { minerals }\end{array}$ & $\mathrm{NI}$ & Chromatography \\
\hline Boris et $\mathrm{al}^{27}$ & Fish oil & $\begin{array}{l}900 \mathrm{mg} \text { DHA } \\
+1300 \mathrm{mg} \text { EPA/ } \\
\text { day }\end{array}$ & Olive oil & $\mathrm{NI}$ & Chromatography \\
\hline Bortolozo et $\mathrm{al}^{2}$ & Fish oil & $\begin{array}{l}315 \mathrm{mg} \text { DHA } \\
+80 \mathrm{mg} \\
\text { EPA/day }\end{array}$ & Maize starch & $\begin{array}{l}\text { 24h reminder on } \\
\text { alternate days of } \\
\text { the week, includ- } \\
\text { ing a weekend day }\end{array}$ & Chromatography \\
\hline Dunstan et $\mathrm{al}^{20}$ & Fish oil & $\begin{array}{l}2200 \mathrm{mg} \text { DHA } \\
+1100 \mathrm{mg} \text { EPA / } \\
\text { day }\end{array}$ & Olive oil & $\mathrm{NI}$ & Chromatography \\
\hline Fidler et al ${ }^{17}$ & $\begin{array}{l}\text { Oil rich in DHA } \\
(\mathrm{DHASCO})^{1}\end{array}$ & 200 mg DHA / day & $\begin{array}{l}\text { Mixture of soy- } \\
\text { bean and corn oils }\end{array}$ & 7-day food record & Chromatography \\
\hline Francois et $\mathrm{al}^{36}$ & Linseed oil & $10.7 \mathrm{~g} \mathrm{ALA}$ & $\mathrm{NI}$ & Food survey & Chromatography \\
\hline Gaete and Atalah ${ }^{1}$ & Food education & $\mathrm{NI}$ & $\mathrm{NI}$ & Food survey & Chromatography \\
\hline Gaete et $\mathrm{al}^{31}$ & $\begin{array}{l}\text { Canned fish(horse } \\
\text { mackerel) }\end{array}$ & $\begin{array}{l}160 \mathrm{~g} \text { fish } 2 \text { times } \\
\text { a week }\end{array}$ & Regular food & Food survey & Chromatography \\
\hline van Goor et al ${ }^{39}$ & $\begin{array}{l}\text { DHA + ARA cap- } \\
\text { sules, DHA } \\
\text { capsules }\end{array}$ & $\begin{array}{l}\text { Group } \\
1=220 \mathrm{mg} \mathrm{DHA} \\
+36 \mathrm{mg} \text { EPA } \\
+220 \mathrm{mg} \text { ARA } \\
+7 \mathrm{mg} \text { ALA }+46 \\
\text { LA } \\
\text { Group } \\
2=220 \mathrm{mg} \text { DHA, } \\
34 \mathrm{mg} \text { EPA }+15 \\
\text { mg ARA }+32 \\
\text { mg ALA }+274 \mathrm{LA}\end{array}$ & Soybean oil & $\mathrm{NI}$ & Chromatography \\
\hline Hawkes et al ${ }^{40}$ & Tuna oil & $\begin{array}{l}\text { Group } \\
1=300 \mathrm{mg} \text { DHA } \\
+70 \mathrm{mg} \text { EPA/day } \\
\text { Group } \\
2=600 \mathrm{mg} \mathrm{DHA} \\
+140 \mathrm{mg} \mathrm{EPA} / \\
\text { day }\end{array}$ & Sunflower seed oil & $\mathrm{NI}$ & Chromatography \\
\hline $\begin{array}{l}\text { Imhoff-Kunsch } \\
\text { et } \mathrm{al}^{13}\end{array}$ & Seaweed oil & $400 \mathrm{mg} \mathrm{DHA}$ & Olive oil & Food survey & Chromatography \\
\hline
\end{tabular}


Table 3 (Continued)

\begin{tabular}{|c|c|c|c|c|c|}
\hline Authors & $\begin{array}{l}\text { Type of } \\
\text { supplement/food } \\
\text { used as source of } \\
\text { omega-3 }\end{array}$ & Amount offered & Placebo/control & $\begin{array}{l}\text { Method used to } \\
\text { evaluate omega-3 } \\
\text { intake }\end{array}$ & $\begin{array}{l}\text { Method to evalu- } \\
\text { ate the amount } \\
\text { of omega-3 pre- } \\
\text { sent in HM }\end{array}$ \\
\hline Jensen et al $^{18}$ & $\begin{array}{l}\text { Group 1: supple- } \\
\text { ment made from } \\
\text { algae with a high } \\
\text { content of DHA } \\
\text { Group 2: eggs with } \\
\text { a high content of } \\
\text { DHA } \\
\text { Group 3: fish oil }\end{array}$ & $\begin{array}{l}\text { Group 1: } 230 \mathrm{mg} \\
\text { DHA/day } \\
\text { Group 2: } 170 \mathrm{mg} \\
\text { DHA/day } \\
\text { Group 3: } 260 \mathrm{mg} \\
\text { DHA/day }\end{array}$ & Eggs & $\mathrm{NI}$ & Chromatography \\
\hline Marc et $\mathrm{al}^{32}$ & $\begin{array}{l}\text { Oil rich in DHA } \\
(\mathrm{DHASCO})^{*}\end{array}$ & $\begin{array}{l}1200 \mathrm{mg} \mathrm{DHA} / \\
\text { day }\end{array}$ & No intervention & Food survey & Chromatography \\
\hline Olafsdottir et al ${ }^{41}$ & Cod liver oil & $\begin{array}{l}1107 \mathrm{mg} \text { DHA } \\
+783 \mathrm{mg} \text { EPA/ } \\
\text { day }\end{array}$ & No intervention & $\begin{array}{l}\text { 24h reminder }+ \\
\text { additional ques- } \\
\text { tions about fish } \\
\text { consumption }\end{array}$ & Chromatography \\
\hline Patin et $\mathrm{al}^{35}$ & Fried sardines & $\begin{array}{l}4 \mathrm{~kg} \text { of sardines } \\
(2 \mathrm{~kg} \text { on day } 0 \text { and } \\
2 \mathrm{~kg} \text { on the } 15 \mathrm{th} \\
\text { day) }\end{array}$ & $\begin{array}{l}\text { The study had no } \\
\text { control group }\end{array}$ & $24 \mathrm{~h}$ reminder & Chromatography \\
\hline Authors & $\begin{array}{l}\text { Type of supple- } \\
\text { ment/food used as } \\
\text { source ofomega-3 }\end{array}$ & Value offered & Placebo/control & $\begin{array}{l}\text { Method used to } \\
\text { evaluate omega-3 } \\
\text { intake }\end{array}$ & $\begin{array}{l}\text { Method to evalu- } \\
\text { ate the amount of } \\
\text { omega-3 present } \\
\text { in HM }\end{array}$ \\
\hline Ribeiro et $\mathrm{al}^{30}$ & Fish oil & $0.72 \mathrm{~g} \mathrm{\omega} /$ day & Primroseoil & $24 \mathrm{~h}$ reminder & Chromatography \\
\hline Sherry et al ${ }^{42}$ & Oil rich in $\mathrm{DHA}$ & $\begin{array}{l}\text { Group 1: } 200 \mathrm{mg} \\
\text { DHA/day } \\
\text { Group 2: } 400 \mathrm{mg} \\
\text { DHA/day }\end{array}$ & $\mathrm{NI}$ & $\begin{array}{l}\text { Food survey and 3- } \\
\text { day food records }\end{array}$ & Chromatography \\
\hline Smit et $\mathrm{al}^{37}$ & $\begin{array}{l}\text { Oil rich in ARA and } \\
\text { DHA }\end{array}$ & $\begin{array}{l}\text { Group 1: } 300 \mathrm{mg} \\
\text { ARA/day } \\
\text { Group 2: } 300 \mathrm{mg} \\
\text { ARA + } 110 \mathrm{mg} \\
\text { EPA and } 400 \mathrm{mg} \\
\text { DHA/day }\end{array}$ & No intervention & $\mathrm{NI}$ & Chromatography \\
\hline Smithers et $\mathrm{al}^{43}$ & Tuna oil & $\begin{array}{l}900 \mathrm{mg} \text { DHA } \\
+195 \mathrm{mg} \text { EPA } \\
+54 \mathrm{mg} \text { ARA / } \\
\text { day }\end{array}$ & Soybean oil & $\mathrm{NI}$ & Chromatography \\
\hline 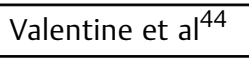 & Seaweed oil & $1000 \mathrm{mg} \mathrm{DHA}$ & Soybean oil & 3-day foodrecords & Chromatography \\
\hline Weseler et $\mathrm{al}^{45}$ & $\begin{array}{l}\text { Milk drink made } \\
\text { from powdered } \\
\text { milk enriched with } \\
\text { LCPUFAs }\end{array}$ & $\begin{array}{l}\text { Group 1: } 200 \mathrm{mg} \\
\text { ARA/day } \\
\text { Group 2: } 400 \mathrm{mg} \\
\text { ARA/day } \\
\text { Group 3: } 320 \mathrm{mg} \\
\text { DHA + } 80 \mathrm{mg} \\
\text { EPA }\end{array}$ & Powdered milk & $\mathrm{NI}$ & Chromatography \\
\hline
\end{tabular}

Abbreviation: ALA, $\alpha$ - linolenic acid; ARA, arachidonic acid; DHA, docosahexaenoic acid; EPA, eicosapentaenoic acid; FOS, fructooligosaccharide; HM, human milk; LA, linoleic acid; LCPUFAs, long chain polyunsaturated fatty acids; NI, Not informed.

${ }^{*}$ DHASCO is an oil derived from a single-celled alga, mainly containing DHA, myristic, palmitic and oleic acids.

fatty acids in the form of linoleic acids (LAs) and $\alpha$ - linolenic acids (ALAs) are activated in the forms known as ketoacyl-CoA, and then used for the conversion of long chain polyunsaturated fatty acids and other polyunsaturated products, such as those derived from the series of desaturation and elongation reactions that are particularly active in the liver and, to a lesser extent, in other tissues. ${ }^{8}$
Linoleic and ALA fatty acids need to be ingested through food, since the human body does not have enzymes to synthesize them. Some vegetables synthesize them and are, therefore, an abundant source of these fatty acids, as well as the products derived from these vegetables. Omega- 3 fatty acids (DHA and EPA) can be synthesized by the human body to a certain level, albeit a very limited one. The consumption of omega- 3 sources 
through diet can be done by ingesting fish or fish oils, and foods enriched or fortified with these important fatty acids. ${ }^{9}$

Although ALA in humans is converted to EPA and DHA, the exact percentage of this conversion is unknown, but it is estimated to be low (5\% EPA and 0.5\% DHA). ${ }^{10,11}$ Due to their enzymatic immaturity, children and especially neonates cannot convert all the DHA required for their development from ALA. ${ }^{12}$ Therefore, feeding in the gestational period is of great importance as it determines the type of fatty acid that will accumulate in the fetal tissue. The essential fatty acids are transferred through the placenta, and in the third gestational trimester are deposited in the brain and retina of the fetus. It should be noted that the fetus withdraws a total of 50 to $75 \mathrm{mg}$ of polyunsaturated fatty acids from the mother, most of them being DHA. ${ }^{13-16}$

Numerous studies have been conducted to evaluate the effects of the supplementation of omega- 3 and its metabolites in pregnancy and puerperium on the composition of HM. This is due to the synthesis of DHA probably occurring minimally in the mammary gland ${ }^{6,17,18}$ as well as due to the role that this polyunsaturated fatty acid plays on visual acuity, cognition and in the formation of the nervous tissue of the newborn. ${ }^{19}$

Although supplementation appears to be the most reliable medium for increasing omega-3 levels in HM, there are numerous differences among the studies evaluated in relation to the following parameters: sample size, study design, timing of omega-3 supplementation (gestation and/or lactation), type of supplementation (fish oil, in natura fish consumption), and amount and type of omega-3 offered (EPA and/or DHA).

Regarding the diversity of the countries where the studies selected for this systematic review were performed, it is worth noting that the consumption of omega- 3 rich foods in Western countries is well below that of other countries. ${ }^{20}$ In the United States, the intake of omega- 3 and its metabolites (DHA and EPA) was estimated at 1.6 and $0.1-0.2 \mathrm{~g} /$ day respectively, and the dietary ratio between omega- 6 and omega-3 was $\approx 9.8: 1 .^{21}$ A study with Canadian pregnant women showed that the average daily intake of omega- 3 and DHA was 1.45 and 0.082 g/day respectively. ${ }^{22}$ Populations living in coastal countries, such as Japan and Norway, where fish are widely consumed, have a higher dietary intake of omega-3 (>1 g/day), and consequently, high concentrations of DHA in their breast milk. ${ }^{20,23,24}$ Although there is no official dietary recommendation for EPA and DHA in the US, several expert groups suggest a DHA intake of at least 200 $\mathrm{mg} /$ day, which may reach $1,000 \mathrm{mg}$ DHA/day for pregnant and lactating women, and 1.4-2.7 g of omega-3, and suggest the omega-6/omega- 3 ratio of $\approx 2-5: 1 .^{21,25}$

Corroborating the above recommendations, the consensus published by Koletzko et $\mathrm{al}^{26}$ states that an average intake of at least $200 \mathrm{mg}$ of DHA per day is advisable; it also states that consumption of up to $1 \mathrm{~g}$ of DHA or 2 to $7 \mathrm{~g}$ of omega-3 per day is safe. This amount can be achieved by consuming one to two servings of fish per week, including fatty fish such as herring, mackerel and salmon. However, it is known that the consumption of fish can contribute significantly to the exposure to contaminants such as methylmercury, which is particularly toxic to the developing brain and possibly harmful to infant growth. To decrease the amounts of methylmercury in the body, one should reduce the intake of contaminated foods during the pregestational and gestational periods. The fish with the highest levels of methylmercury are predatory fish such as marlin, pike, swordfish and shark. However, after an extensive literature review, the consensus points out that the beneficial effects of regular consumption of fish sources of DHA during pregnancy appear to overcome the potential drawbacks of the increased intake of contaminants.

Regarding the period of supplementation, the selected studies presented different time periods (pregnant and/or nursing) when omega-3 supplementation was performed and measured, which may partially justify the differences in the results we found. On this issue, in their randomized clinical trial, Boris et $\mathrm{al}^{27}$ evaluated two hypotheses, namely: 1) whether omega-3 supplementation during pregnancy increased omega- 3 levels at the beginning of breastfeeding; and 2) whether the continuation of supplementation after delivery was necessary to sustain the long-term increase in omega-3 levels. There was a marked drop in omega-3 levels in the group that stopped supplementation during the puerperium. Such a decrease in the concentration of DHA in breast milk as lactation progresses is corroborated by numerous studies. $^{4,28,29}$ On the other hand, the group that received fish oil during gestation and lactation showed levels of omega- 3 three times higher, and double the levels of DHA. ${ }^{27}$ It is worth mentioning that polyunsaturated fatty acids are deposited in the brain during the last gestational trimester, and that this process continues after delivery. Furthermore, the neurological development continues during the first years of life. ${ }^{27}$ The results found by Ribeiro et $\mathrm{al}^{30}$ also demonstrated that supplementation with fish oil limited to pregnancy was not as effective as supplementation during pregnancy and lactation. Therefore, supplementation during pregnancy and lactation is recommended by numerous studies. ${ }^{20,30,31}$

Important issues to take into account in these studies are the type of omega- 3 source and the quantity that was supplied. It was observed that most of the selected studies used fish oil to increase the consumption of omega-3; however, some studies have used the supply of fresh food, fortified drinks and food education techniques. The use of fish oil has benefits, but it can lead to low compliance due to its adverse effects, such as fish flavor eructation, digestive discomfort and night sweats. ${ }^{5,32}$ The randomized double blind clinical trial conducted by Dunstan et $\mathrm{al}^{20}$ aimed to evaluate the effects of fish oil supplementation during pregnancy on the composition of $\mathrm{HM}$ and on the development of the infant in the first year of life. The concentration of fatty acids in the milk was analyzed on the third day, sixth week and sixth month after delivery. It was observed that women who received fish oil had a higher concentration of EPA and DHA in the milk on the third day and the sixth week after delivery.

Regarding the consumption of fish, the study by Henderson et $\mathrm{al}^{33}$ demonstrated that ingesting 100-120 g of sardines 2 to 3 times a week resulted in increased levels of fatty acids without the need for fish oil. Harris et $\mathrm{al}^{34}$ disagreed 


\begin{tabular}{|c|c|c|c|c|c|c|}
\hline $\begin{array}{l}\frac{\underline{y}}{\bar{z}} \\
\ddot{u}\end{array}$ & 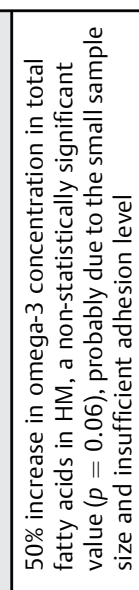 & 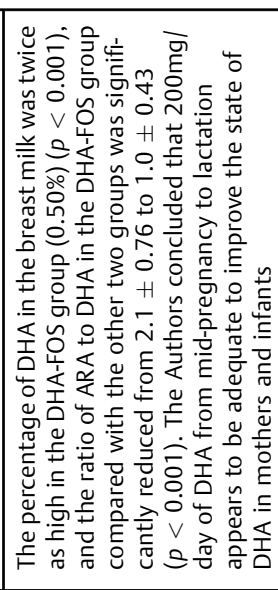 & 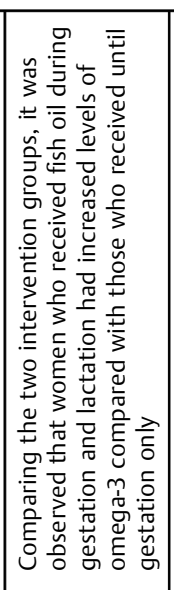 & 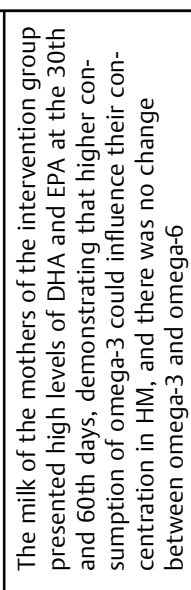 & 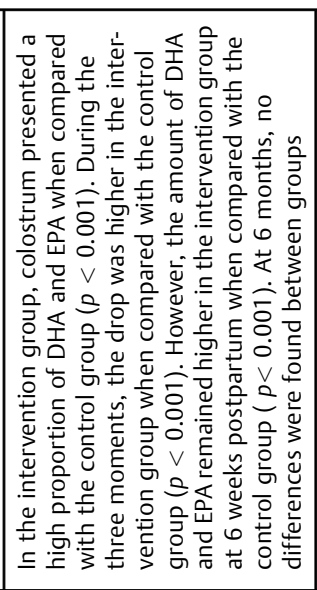 & 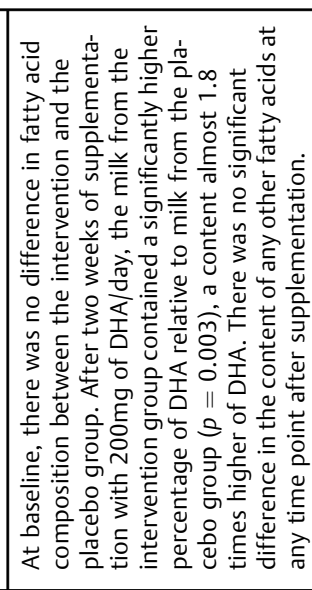 \\
\hline 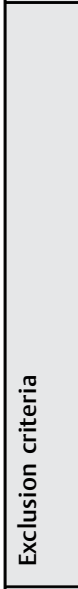 & $\bar{z}$ & 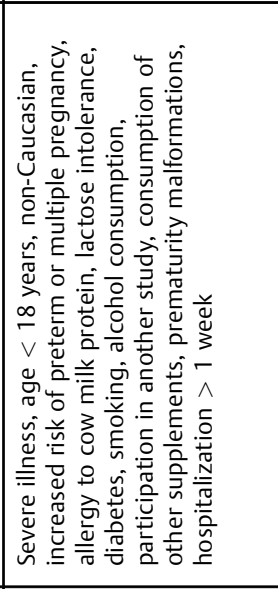 & $\bar{z}$ & $\bar{z}$ & 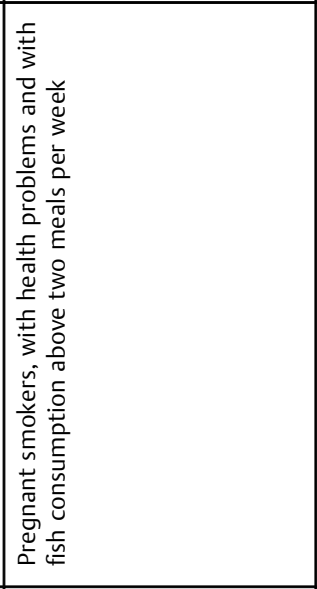 & $\bar{z}$ \\
\hline & 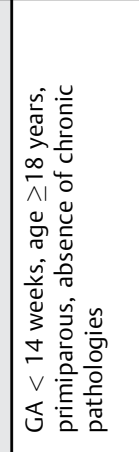 & 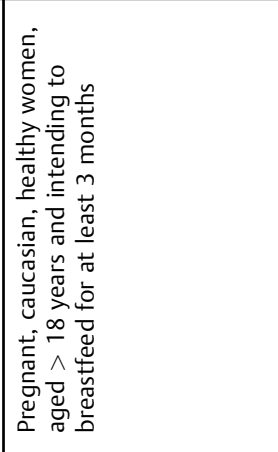 & $\bar{z}$ & 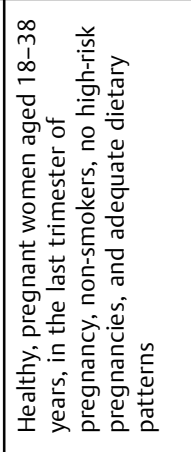 & 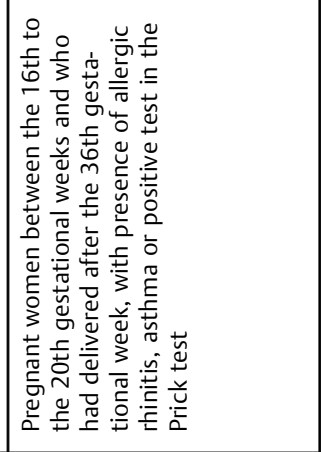 & 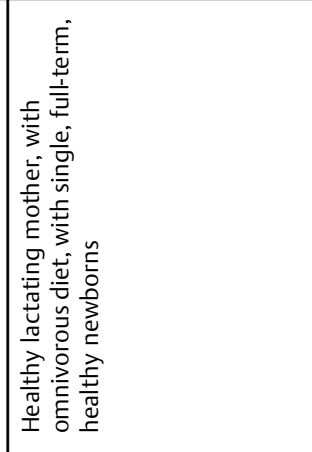 \\
\hline 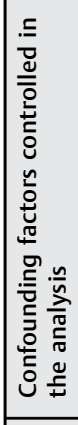 & 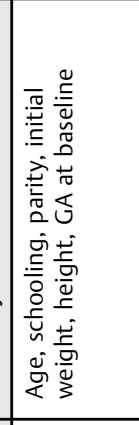 & 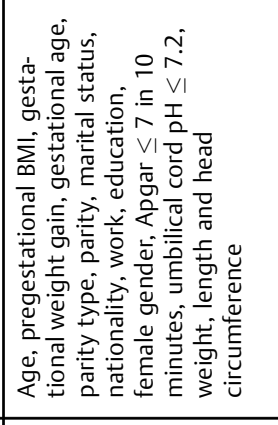 & $\bar{z}$ & 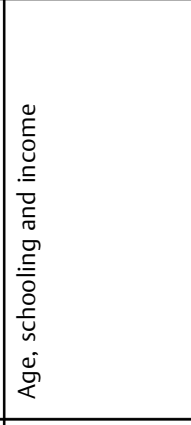 & 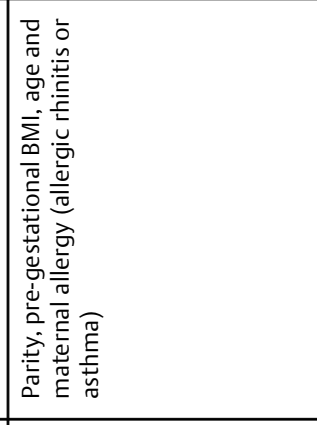 & 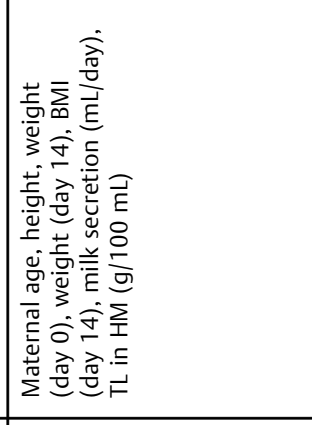 \\
\hline 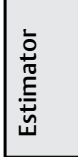 & 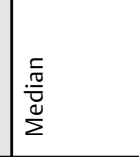 & 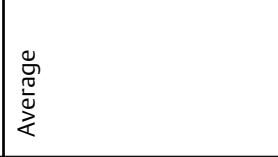 & 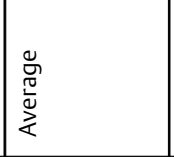 & 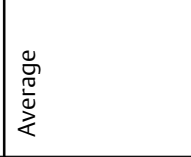 & 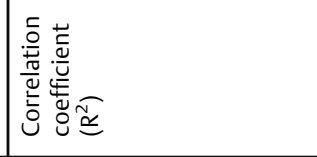 & 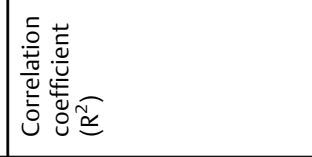 \\
\hline 遟 & 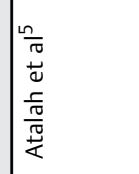 & 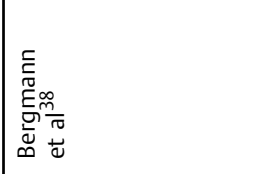 & 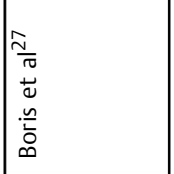 & 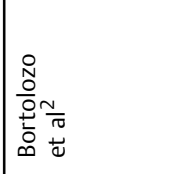 & 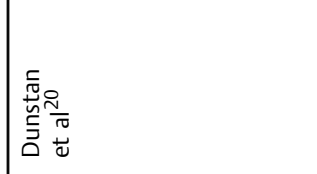 & 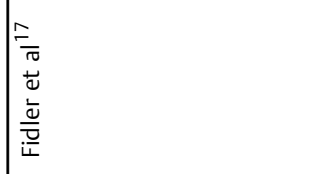 \\
\hline
\end{tabular}




\begin{tabular}{|c|c|c|c|c|c|c|c|c|}
\hline 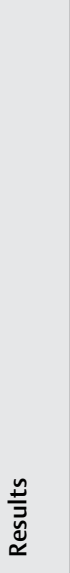 & 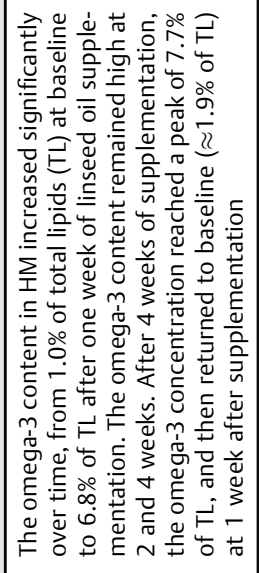 & 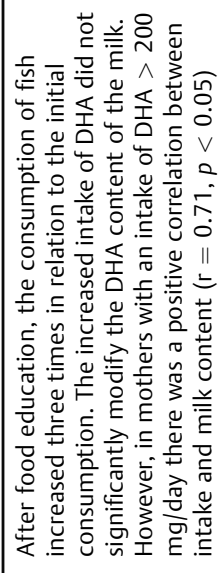 & 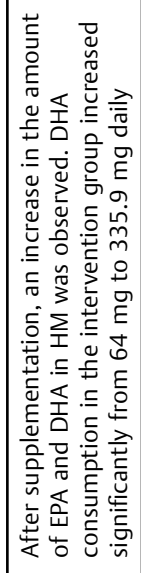 & 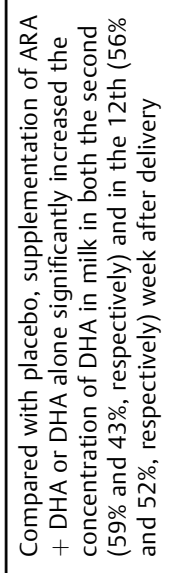 & 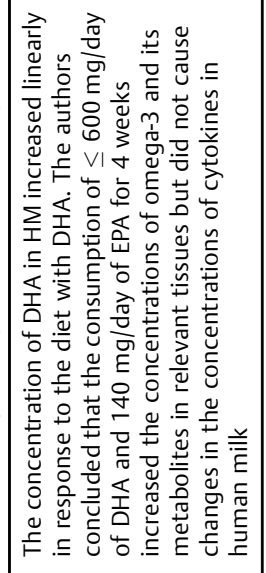 & 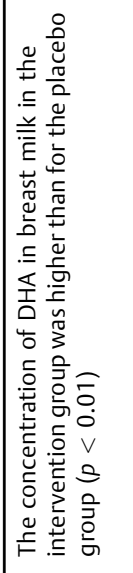 & 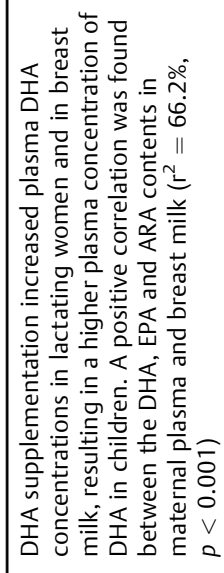 & 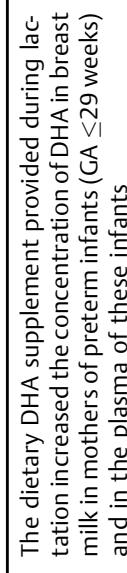 \\
\hline 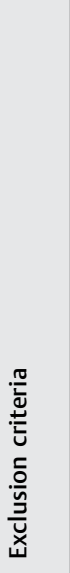 & $\bar{z}$ & 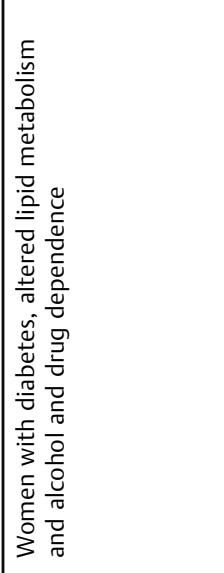 & 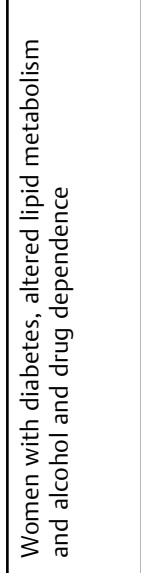 & 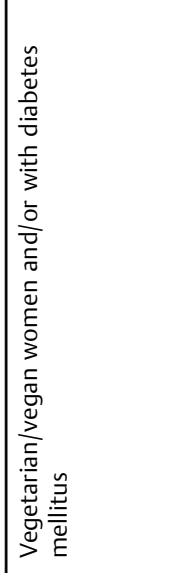 & 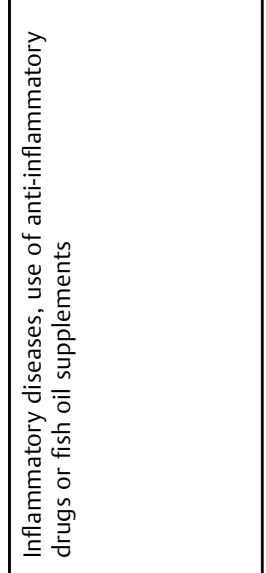 & 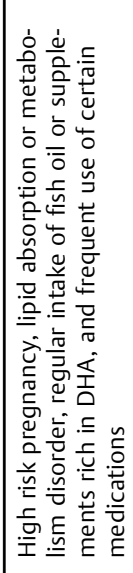 & 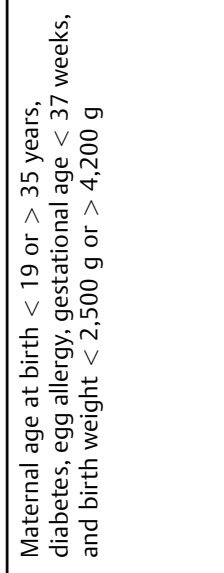 & 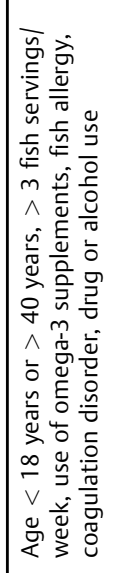 \\
\hline 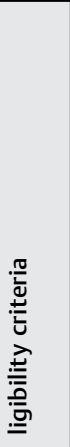 & 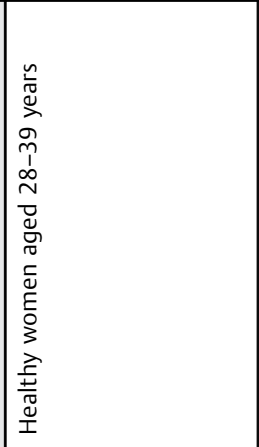 & 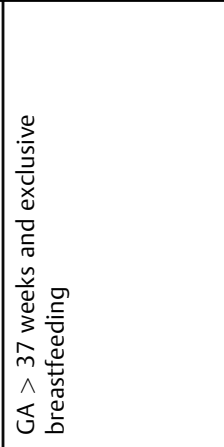 & 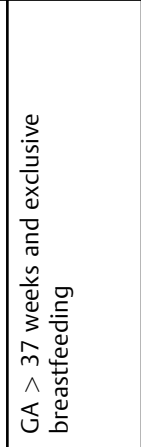 & 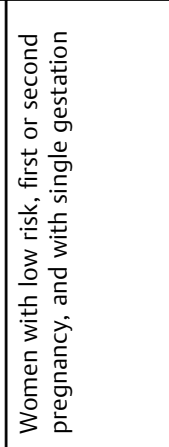 & 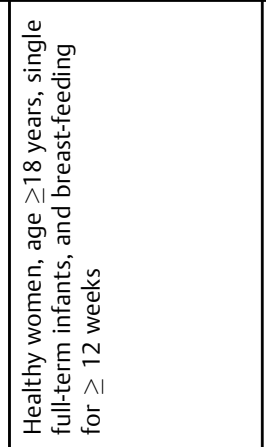 & 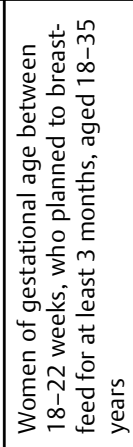 & 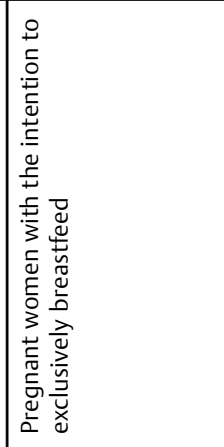 & 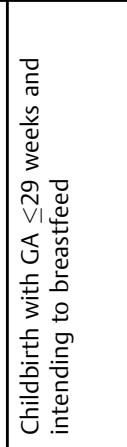 \\
\hline 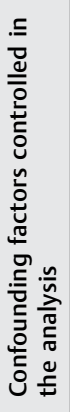 & $\bar{z}$ & 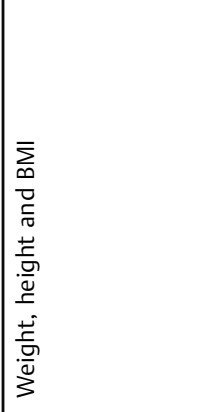 & 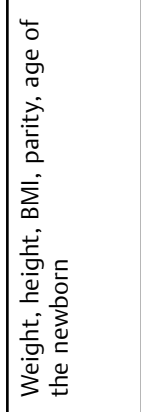 & 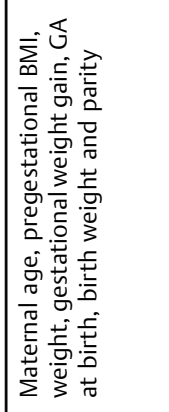 & 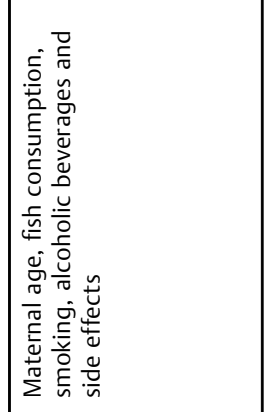 & 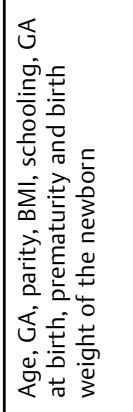 & 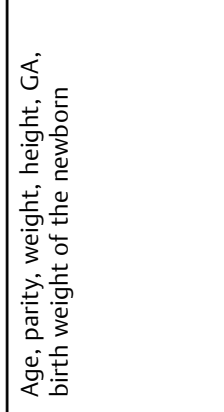 & 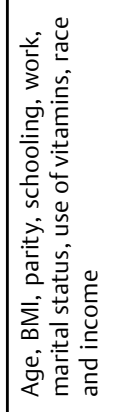 \\
\hline 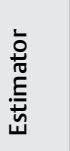 & 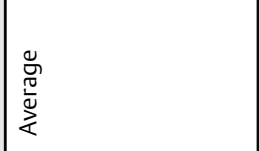 & $\begin{array}{l}\frac{.}{.0} \\
\frac{.0}{0} \\
\stackrel{0}{\Sigma}\end{array}$ & 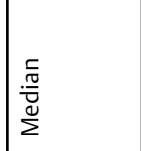 & 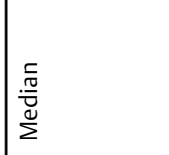 & 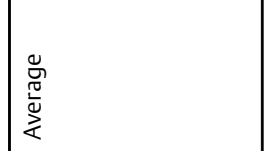 & 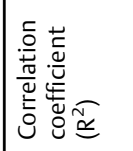 & 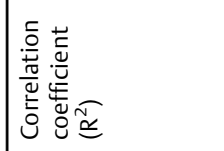 & 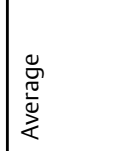 \\
\hline $\begin{array}{l}\frac{n}{0} \\
\frac{c}{2} \\
\frac{1}{\alpha}\end{array}$ & 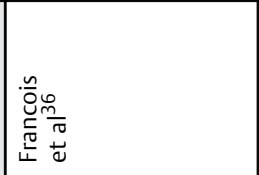 & 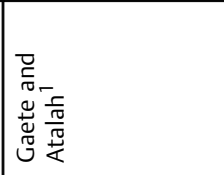 & 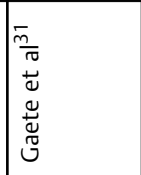 & 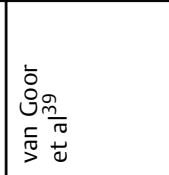 & 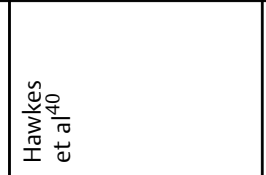 & 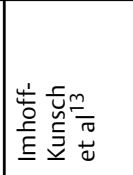 & 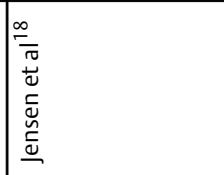 & 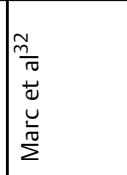 \\
\hline
\end{tabular}



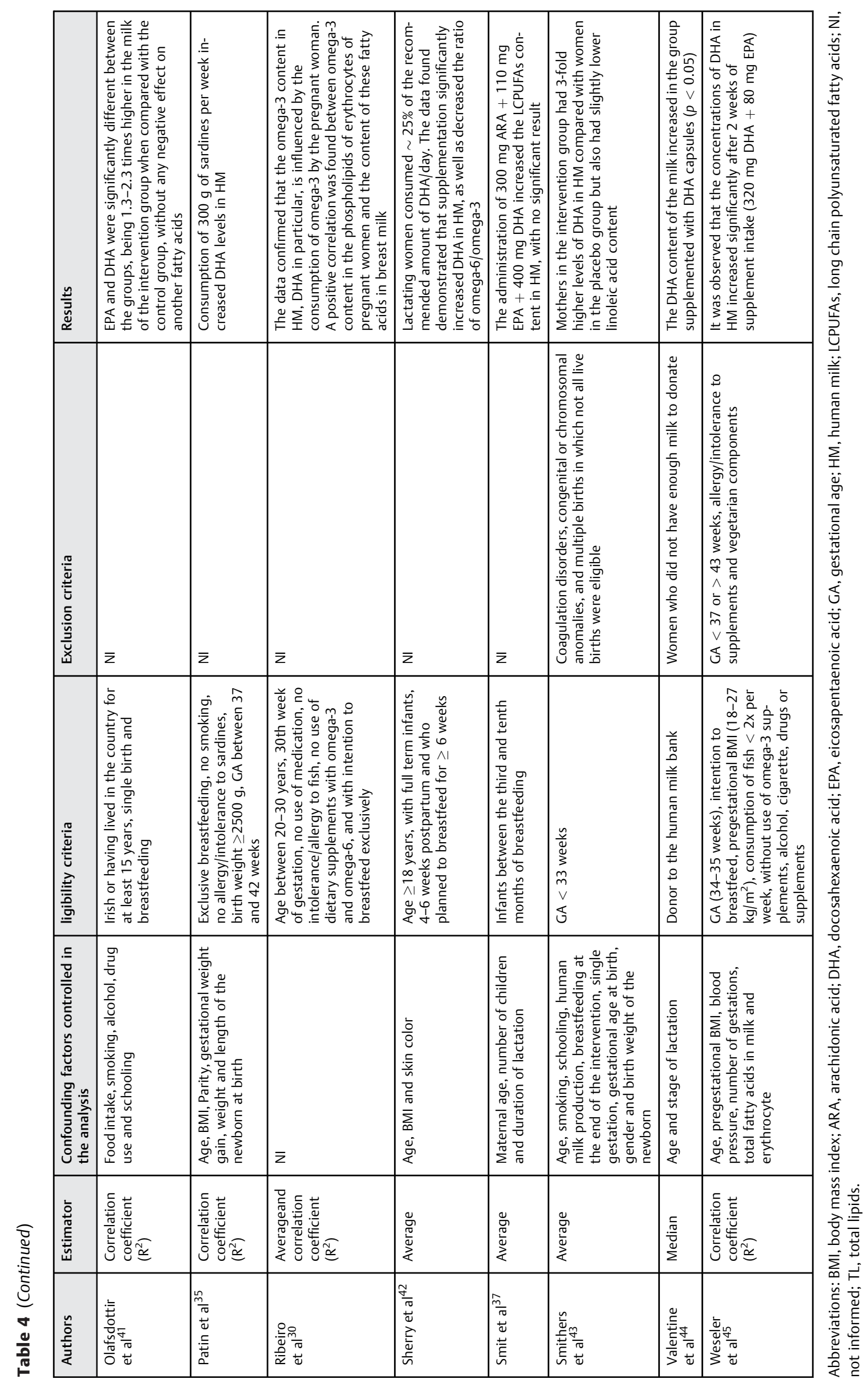
with this, and have observed that in order to increase 0.5 to $1 \mathrm{~g}$ of DHA in breast milk, it is necessary to consume 350 $750 \mathrm{~g}$ of $1 \%$ fat or $75-150 \mathrm{~g}$ of $10 \%$ fish fat. In the study by Patin et al, ${ }^{35}$ it was observed that the levels of DHA in HM increased with the ingestion of $300 \mathrm{~g}$ of sardines per week, with $5 \%$ fat, without the need to use fish oil supplementation. This study recommended the consumption of fish two to three times per week during gestation.

Gaete and Atalah ${ }^{1}$ conducted a prospective study with 26 pregnant women, which consisted of an educational feeding strategy to recommend individual consumption of different preparations based on marine foods. A guide with information on the importance of maternal lactation to the newborn, and on the importance of fish consumption by the mother to increase DHA levels was also distributed. The strategy of food education is considered an important intervention to raise awareness about the need for fish consumption during the gestational and puerperal period.

The study by Atalah et $\mathrm{al}^{5}$ aimed to evaluate the effects of the introduction of omega- 3 fortified milk beverages (DHA and EPA) during gestation on the composition of HM and red blood cells. One-hundred and seventy-five women from the intervention group and 177 from the control group were evaluated in the clinical trial. The pregnant women were evaluated at three moments of the pregnancy and once after delivery to evaluate the consumption near the date of the interview. The evaluation of milk composition was performed in only 16 women, and a 50\% increase in omega-3 in breast milk was observed. However, there was no statistical difference between the evaluated groups in relation to the amount of EPA and DHA, probably due to the small sample size.

Regarding the type of omega-3 offered, seven studies offered DHA and EPA, seven offered DHA only, and three offered DHA, arachidonic acid (ARA) and EPA. It was observed that the amount of DHA was always higher than that of EPA, probably because of its important role on the nervous system, cognition and vision. It is worth noting that there is no consensus regarding the optimal levels of DHA consumption at different stages of life. However, most technical groups recommend around 200 to $500 \mathrm{mg}$ /day in the adult population, and, during gestation, it is recommended to consume fish between two to three times per week. ${ }^{5}$

There are numerous factors that contribute to the variability of EPA and DHA content in breast milk, such as lactation stage, gestational age, and maternal nutritional status. What is verified is that certain selected studies ${ }^{20,27,30,36}$ did not control the analyses for important confounding factors, such as food consumption. Therefore, estimates of association may be compromised by the fact that certain studies did not quantify follow-up losses, but also because they did not control important confounding factors.

All selected articles showed the importance of supplementation of omega-3 in different forms (capsules, dairy drinks, strategy for feeding education, consumption of fish) on the nutritional composition of HM in the gestational and/ or puerperal periods. However, four studies ${ }^{1,5,27,37}$ did not reach statistical significance. This can be partially explained by the sample size, which can reduce the strength of the study to elucidate possible associations, possible adhesion reduction in relation to the intake of supplements and the food education practices employed, as well as the follow-up losses, which may cause a decrease in the validity of the results.

\section{Conclusion}

Although the studies were disparate in several methodological aspects, the importance of omega-3 supplementation in pregnancy and/or the puerperium, especially DHA, as well as the safety of its supplementation were observed with the data from the studies that composed this systematic review. However, it is of great importance that further studies be conducted to establish the adequate amount of omega-3s and their metabolites during gestation and lactation that will bring benefit to newborns.

\section{References}

1 Gaete GM, Atalah SE. Niveles de LC-PUFA n-3 en la leche materna después de incentivar el consumo de alimentos marinos. Rev Chil Pediatr 2003;74(02):158-165

2 Bortolozo EAFQ, Sauer E, Santos MS, et al. Supplementation with the omega-3 docosahexaenoic acid: influence on the lipid composition and fatty acid profile of HM. Rev Nutr 2013;26(01): 27-36

3 Ballard O, Morrow AL. Human milk composition: nutrients and bioactive factors. Pediatr Clin North Am 2013;60(01):49-74

4 Nishimura RY, Barbieiri P, Castro GS, Jordão AA Jr, Perdoná GdaS, Sartorelli DS. Dietary polyunsaturated fatty acid intake during late pregnancy affects fatty acid composition of mature breast milk. Nutrition 2014;30(06):685-689

5 Atalah SE, Araya BM, Rosselot PG, et al. Consumption of a DHAenriched milk drink by pregnant and lactating women, on the fatty acid composition of red blood cells, breast milk, and in the newborn. Arch Latinoam Nutr 2009;59(03):271-277

6 Innis SM. Human milk: maternal dietary lipids and infant development. Proc Nutr Soc 2007;66(03):397-404

7 Moher D, Liberati A, Tetzlaff J, Altman DG; PRISMA Group. Preferred reporting items for systematic reviews and meta-analyses: the PRISMA statement. LoS Med 2009;6(07):e1000097

8 Williams CM, Burdge G. Long-chain n-3 PUFA: plant v. marine sources. Proc Nutr Soc 2006;65(01):42-50

9 Holub BJ. Clinical nutrition: 4. Omega-3 fatty acids in cardiovascular care. CMAJ 2002;166(05):608-615

10 Burdge GC. Metabolism of alpha-linolenic acid in humans. Prostaglandins Leukot Essent Fatty Acids 2006;75(03):161-168

11 Kus MM, Mancini-Filho J. Ácidos graxos: eicosapentaenóico (EPA) e docosahexaenóico (DHA). São Paulo: ILSI Brasil; 2010

12 Agostoni C. Role of long-chain polyunsaturated fatty acids in the first year of life. J Pediatr Gastroenterol Nutr 2008;47(Suppl 2): S41-S44

13 Imhoff-Kunsch B, Stein AD, Martorell R, Parra-Cabrera S, Romieu I, Ramakrishnan U. Prenatal docosahexaenoic acid supplementation and infant morbidity: randomized controlled trial. Pediatrics 2011;128(03):e505-e512

14 Carlson SE, Colombo J, Gajewski BJ, et al. DHA supplementation and pregnancy outcomes. Am J Clin Nutr 2013;97(04):808-815

15 Rogers LK, Valentine CJ, Keim SA. DHA supplementation: current implications in pregnancy and childhood. Pharmacol Res 2013; 70(01):13-19 
16 Swanson D, Block R, Mousa SA. Omega-3 fatty acids EPA and DHA: health benefits throughout life. Adv Nutr 2012;3(01):1-7

17 Fidler N, Sauerwald T, Pohl A, Demmelmair H, Koletzko B. Docosahexaenoic acid transfer into human milk after dietary supplementation: a randomized clinical trial. J Lipid Res 2000; 41(09):1376-1383

18 Jensen CL, Maude M, Anderson RE, Heird WC. Effect of docosahexaenoic acid supplementation of lactating women on the fatty acid composition of breast milk lipids and maternal and infant plasma phospholipids. Am J Clin Nutr 2000;71(1, Suppl):292S-299S

19 Makrides M. Outcomes for mothers and their babies: do n-3 longchain polyunsaturated fatty acids and seafoods make a difference? J Am Diet Assoc 2008;108(10):1622-1626

20 Dunstan JA, Mitoulas LR, Dixon G, et al. The effects of fish oil supplementation in pregnancy on breast milk fatty acid composition over the course of lactation: a randomized controlled trial. Pediatr Res 2007;62(06):689-694

21 Kris-Etherton PM, Taylor DS, Yu-Poth S, et al. Polyunsaturated fatty acids in the food chain in the United States. Am J Clin Nutr 2000;71(1, Suppl)179S-188S

22 Denomme J, Stark KD, Holub BJ. Directly quantitated dietary (n-3) fatty acid intakes of pregnant Canadian women are lower than current dietary recommendations. J Nutr 2005;135(02):206-211

23 Hibbeln JR, Nieminen LR, Blasbalg TL, Riggs JA, Lands WE. Healthy intakes of n-3 and n- 6 fatty acids: estimations considering worldwide diversity. Am J Clin Nutr 2006;83(6, Suppl)1483S-1493S

24 Brenna JT, Varamini B, Jensen RG, Diersen-Schade DA, Boettcher JA, Arterburn LM. Docosahexaenoic and arachidonic acid concentrations in human breast milk worldwide. Am J Clin Nutr 2007;85(06):1457-1464

25 Koletzko B, Lien E, Agostoni C, et al; World Association of Perinatal Medicine Dietary Guidelines Working Group. The roles of longchain polyunsaturated fatty acids in pregnancy, lactation and infancy: review of current knowledge and consensus recommendations. J Perinat Med 2008;36(01):5-14

26 Koletzko B, Cetin I, Brenna JT; Perinatal Lipid Intake Working Group; Child Health Foundation; Diabetic Pregnancy Study Group; European Association of Perinatal Medicine; European Association of Perinatal Medicine; European Society for Clinical Nutrition and Metabolism; European Society for Paediatric Gastroenterology, Hepatology and Nutrition, Committee on Nutrition; International Federation of Placenta Associations; International Society for the Study of Fatty Acids and Lipids. Dietary fat intakes for pregnant and lactating women. Br J Nutr 2007;98(05):873-877

27 Boris J, Jensen B, Salvig JD, Secher NJ, Olsen SF. A randomized controlled trial of the effect of fish oil supplementation in late pregnancy and early lactation on the n-3 fatty acid content in human breast milk. Lipids 2004;39(12):1191-1196

28 Makrides M, Gibson RA. Long-chain polyunsaturated fatty acid requirements during pregnancy and lactation. Am J Clin Nutr 2000;71(1, Suppl)307S-311S

29 Bonham MP, Duffy EM, Wallace JM, et al. Habitual fish consumption does not prevent a decrease in LCPUFA status in pregnant women (the Seychelles Child Development Nutrition Study). Prostaglandins Leukot Essent Fatty Acids 2008;78(06): 343-350

30 Ribeiro P, Carvalho FD, Abreu AdeA, Sant'anna MdeT, Lima RJ, Carvalho PdeO. Effect of fish oil supplementation in pregnancy on the fatty acid composition of erythrocyte phospholipids and breast milk lipids. Int J Food Sci Nutr 2012;63(01):36-40

31 Gaete MG, Atalah ES, Araya JA. Efecto de la suplementación de la dieta de la madre durante la lactancia con ácidos grasos omega 3 en la composición de los lípidos de la leche. Rev Chil Pediatr 2002; 73(03):239-247

32 Marc I, Plourde M, Lucas M, et al. Early docosahexaenoic acid supplementation of mothers during lactation leads to high plasma concentrations in very preterm infants. J Nutr 2011; 141(02):231-236

33 Henderson RA, Jensen RG, Lammi-Keefe CJ, Ferris AM, Dardick KR. Effect of fish oil on the fatty acid composition of human milk and maternal and infant erythrocytes. Lipids 1992;27(11):863-869

34 Harris WS, Connor WE, Lindsey S. Will dietary omega-3 fatty acids change the composition of human milk? Am J Clin Nutr 1984; 40(04):780-785

35 Patin RV, Vítolo MR, Valverde MA, Carvalho PO, Pastore GM, Lopez FA. The influence of sardine consumption on the omega- 3 fatty acid content of mature human milk. J Pediatr (Rio J) 2006;82(01): 63-69

36 Francois CA, Connor SL, Bolewicz LC, Connor WE. Supplementing lactating women with flaxseed oil does not increase docosahexaenoic acid in their milk. Am J Clin Nutr 2003;77(01):226-233

37 Smit EN, Koopmann M, Boersma ER, Muskiet FA. Effect of supplementation of arachidonic acid (AA) or a combination of AA plus docosahexaenoic acid on breastmilk fatty acid composition. Prostaglandins Leukot Essent Fatty Acids 2000;62(06):335-340

38 Bergmann RL, Haschke-Becher E, Klassen-Wigger P, et al. Supplementation with $200 \mathrm{mg} /$ day docosahexaenoic acid from midpregnancy through lactation improves the docosahexaenoic acid status of mothers with a habitually low fish intake and of their infants. Ann Nutr Metab 2008;52(02):157-166

39 van Goor SA, Dijck-Brouwer DA, Hadders-Algra M, et al. Human milk arachidonic acid and docosahexaenoic acid contents increase following supplementation during pregnancy and lactation. Prostaglandins Leukot Essent Fatty Acids 2009; 80(01):65-69

40 Hawkes JS, Bryan DL, Neumann MA, Makrides M, Gibson RA. Transforming growth factor beta in human milk does not change in response to modest intakes of docosahexaenoic acid. Lipids 2001;36(10):1179-1181

41 Olafsdottir AS, Thorsdottir I, Wagner KH, Elmadfa I. Polyunsaturated fatty acids in the diet and breast milk of lactating icelandic women with traditional fish and cod liver oil consumption. Ann Nutr Metab 2006;50(03):270-276

42 Sherry CL, Oliver JS, Marriage BJ. Docosahexaenoic acid supplementation in lactating women increases breast milk and plasma docosahexaenoic acid concentrations and alters infant omega 6:3 fatty acid ratio. Prostaglandins Leukot Essent Fatty Acids 2015; 95:63-69

43 Smithers LG, Markrides M, Gibson RA. Human milk fatty acids from lactating mothers of preterm infants: a study revealing wide intra- and inter-individual variation. Prostaglandins Leukot Essent Fatty Acids 2010;83(01):9-13

44 Valentine CJ, Morrow G, Pennell M, et al. Randomized controlled trial of docosahexaenoic acid supplementation in midwestern $\mathrm{U}$. S. human milk donors. Breastfeed Med 2013;8(01):86-91

45 Weseler AR, Dirix CE, Bruins MJ, Hornstra G. Dietary arachidonic acid dose-dependently increases the arachidonic acid concentration in human milk. J Nutr 2008;138(11):2190-2197 